\title{
Blue fleck corneal iridescence: an occasional feature of Cogan's microcystic corneal dystrophy
}

\author{
ANTHONY J. DARK AND JOHN PROCTOR \\ From the Departments of Ophthalmology and Human Biology, University of Sheffield
}

SUMMARY Flecks consisting of brilliant reflective blue dots and streaks were a prominent biomicroscopical feature in three patients with Cogan's microcystic corneal dystrophy. Two patients required epithelial debridement. We used the material obtained to investigate their histology and ultrastructure. A subepithelial accumulation of basement membrane-like material composed of ultrastructurally fine granules was deposited in alternating layers of compaction and rarefaction. The layers lie mostly parallel to the anterior corneal surface, but in some places they are folded. Such foldings are possibly the sites of multilaminar reflection and constructive interference, giving rise to the blue iridescence.

The geographic or map-like areas which are seen in Cogan's microcystic dystrophy are the essential features of this disorder, although they are sometimes so tenuous that their presence may be initially suspected by deformation of the overlying fluorescein-stained precorneal tear film. Additional findings include greyish-white 'amoeboid' epithelial microcysts and occasional finger-print-like striae.

A 42-year-old man with bilateral chronic ocular irritation was referred by his oculist with the mystifying observation that in addition to map-like opacities there were "blue neon-like lights flickering in the anterior layers of both corneas'. Two female patients, respectively 48 and 56 years old, with similar symptomatology were subsequently observed by one of us. Chronic irritation in 2 of these 3 patients was sufficiently severe to justify debridement of the epithelium, providing material which was examined by light and electron microscopy in an attempt to find the structural basis for the phenomenon.

\section{Patients and methods}

Three patients with chronic ocular discomfort were found to have bilateral Cogan's microcystic dystrophy. Subepithelial flecks consisting of bright blue corneal streaks and dots measuring about 10-100 $\mu \mathrm{m}$ were the prominent biomicroscopic features in both eyes of patient 1 and were unilaterally present in Correspondence to A. J. Dark, MD, Department of Ophthalmology, Royal Hallamshire Hospital, Sheffield S10 2JF. patients 2 and 3 . The blue flecks were bright against a poorly illuminated background, which varied from blue (Figs. 1A and B) to pink (Fig. 1C) depending on whether light was scattered from the iris or fundus. They were present in a variety of forms, including circles and ellipses (sometimes incomplete) and irregular blotches. The flecks remained unchanged in hue and brightness over a wide arc of incident light (as much as $30^{\circ}$ in some instances), though their configuration changed somewhat, some forms expanding or contracting (Figs. 1A, B). In addition some of the streaks disappeared, while others previously invisible now emerged. Rotation of a polarising lens inserted between the cornea and slit-lamp objective caused alterations in brightness but did not produce any change in their colour. The blue figures were generally found at the edge of the map-like opacities, but this was not an invariable relationship. The first patient did not respond to conservative treatment, so that debridement of the corneal epithelium was undertaken on the worse eye; the affected eye of the second patient was treated similarly. In both these patients the epithelium regenerated within a few days, and although there was an immediate subjective improvement which persisted for a month or two, both eyes continue to give rise to irritation some 6 months later. The blue flecks were apparently completely removed by the debridement, since they have not reappeared over a 9-month period. They remained unchanged, however, in the unoperated eyes of patients 1 and 3. 


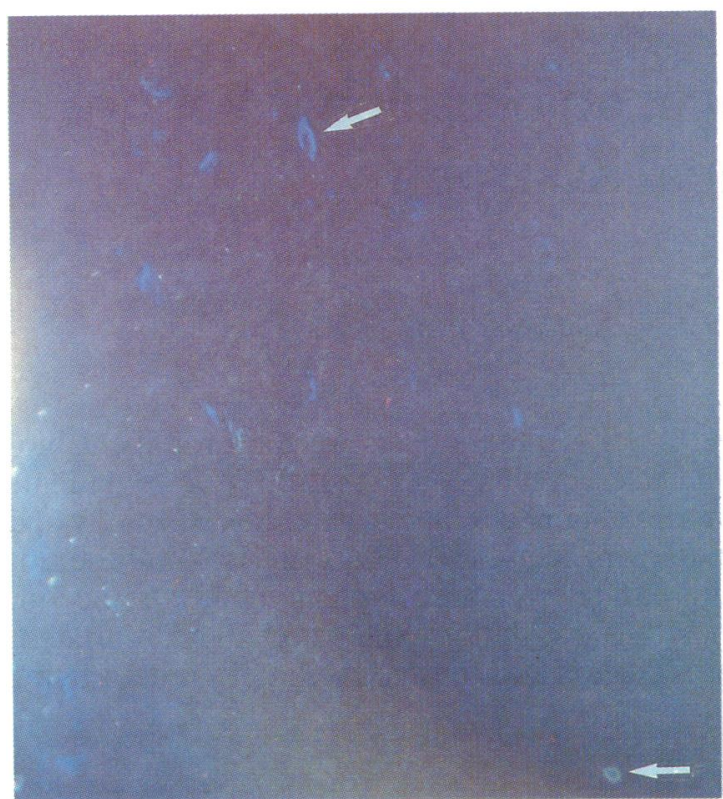

Fig. 1A

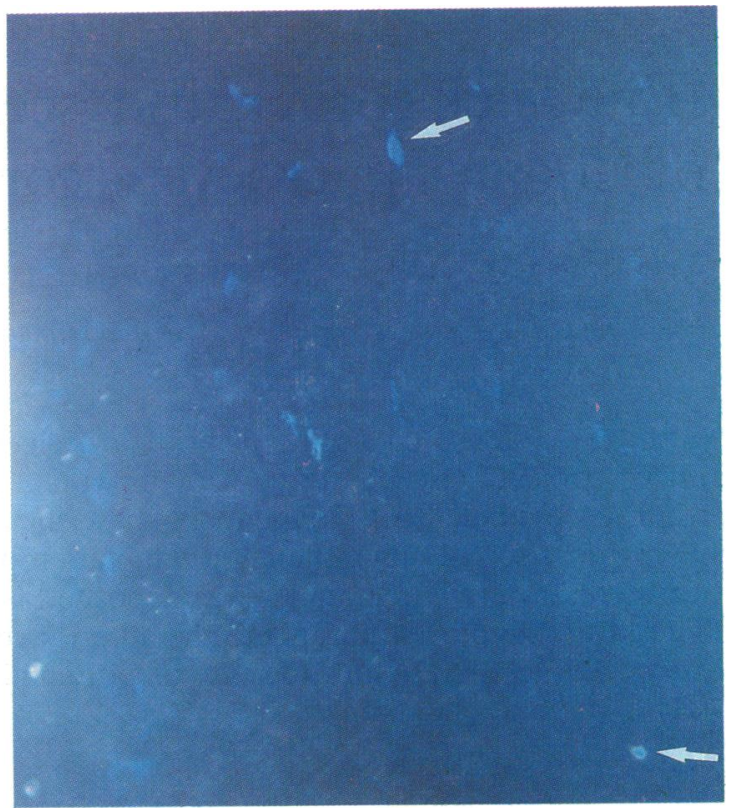

Fig. 1B

Figs. 1A, B Patient 1. Blue flecks in the cornea of a patient with Cogan's microcystic dystrophy. The blue background results from iris retroillumination. These 2 macrophotographs of the same area illustrate the effect of altering the angle of incident light. Circle (arrowed) decreases in diameter, a U-shaped linear figure (arrowed) becomes solid as the angle is changed. $(\times 70)$.

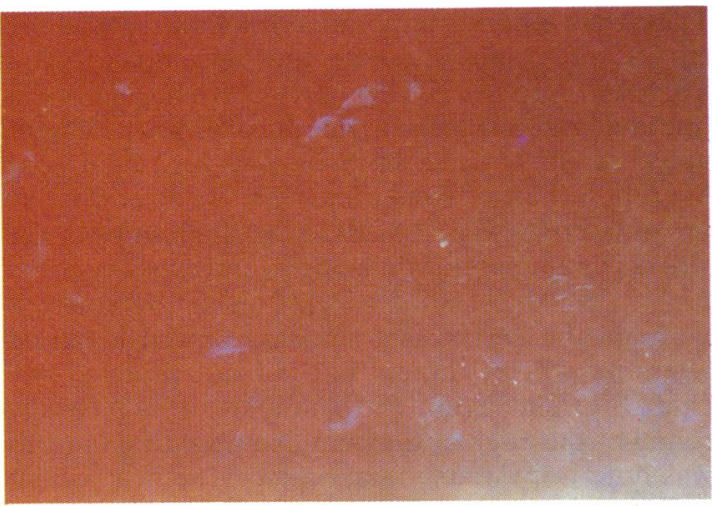

Fig. 1C Patient 2. Blueflecksseenagainstred reflex. $(\times 70)$.

Corneal epithelium from patients 1 and 2 were fixed in $2 \frac{1}{2} \%$ cold glutaraldehyde prior to osmification and embedding in epoxy resin. Orientational sections were stained with methylene blue and examined with the light microscope, while suitably thin sections were stained with uranyl acetate and lead citrate before being submitted to transmission electron microscopy with a Phillips 300 electron microscope.

\section{Results}

LIGHT MICROSCOPY

In methylene blue-stained sections from both patients the corneal epithelium showed no abnormality apart from mild dysplastic changes and the presence of an occasional microcyst. Beneath the epithelium were several layers of basophilic material. These layers, which varied in thickness and density, were mostly arranged parallel to the corneal surface (Fig. 2) but in places they were heaped up into mounds which invaginated the basement membrane into the epithelium. In patient 1, occasional basophilic spherules were embedded in the subepithelial material close to the basement membrane.

\section{TRANSMISSION ELECTRON MICROSCOPY}

The corneal epithelium rested on an intact basement membrane, which had a normal complement of hemidesmosomes. The subepithelial stratified material consisted of myriads of fine amorphous granules, which were closely aggregated in the dense layers but were sparse in the lucent ones. The strata were sharply defined from each other and varied in thickness from $0 \cdot 1$ to $12 \mu \mathrm{m}$ (Fig. 2). In some areas a variety of fibrillar elements were embedded in granular material, usually close to the basement 


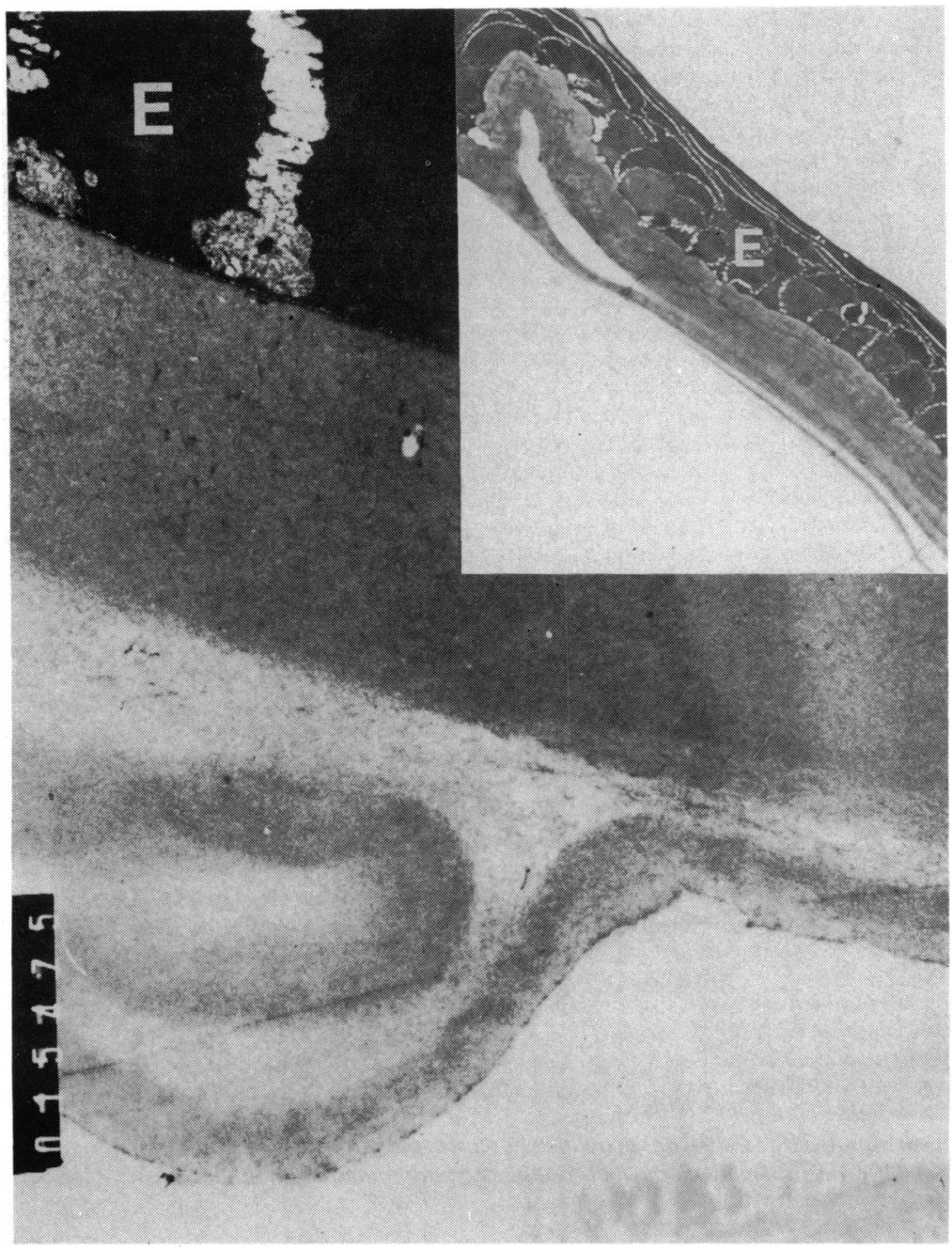

Fig. 2 Patient 2. Corneal epithelium. Elies on multilaminar basement membrane-like material which is composed of fine granules. The granules are compacted and rarefied in alternating layers. The widths of the laminae vary, but some are of the order of the wavelength of light. In places the laminae are rolled up beneath the epithelium. (EM, ×3895). Inset: Methylene blue, $\times 600$.

membrane. A few elongated electron dense cells, possibly keratocytes, were also embedded in the most superficial stratum; they were usually surrounded by a zone of rarefaction. Also in this stratum were occasional electron dense bodies corresponding to the basophilic spheroidal structures seen under the light microscope in patient 1 . These structures varied in size from 130 to $520 \mathrm{~nm}$ and consisted of granules which often formed a compact outer zone surrounding a less densely packed core. No membrane surrounded these bodies, but in many instances a variable number of clear vacuoles were found alongside the granules.

\section{Discussion}

The presence of brilliant blue streaks and dots in the superficial cornea has been noted here as an addi- tional biomicroscopic feature of Cogan's microcystic dystrophy. Rotation of a polarising lens between the cornea and the slit-lamp objective does not alter their colour, so that the presence of a corneal structure acting as a polarising analyser is unlikely. Their constant brightness and hue in spite of wide changes in the angle of incidence suggests that reflectivity rather than refractivity (i.e., iridescence rather than a prismatic effect) would account for their appearance. In contrast they do alter in shape, enlarging and diminishing, and in number, appearing and vanishing, when the angle of incidence is changed. These latter observations would suggest a focal 3-dimensional laminated structural basis for reflection rather than one of interfaces parallel to the corneal surface.

The iridescence of soap bubbles and oil slicks (Meibomian lipid in the precorneal tear film provides a familiar ophthalmic example of the latter) is pro- 


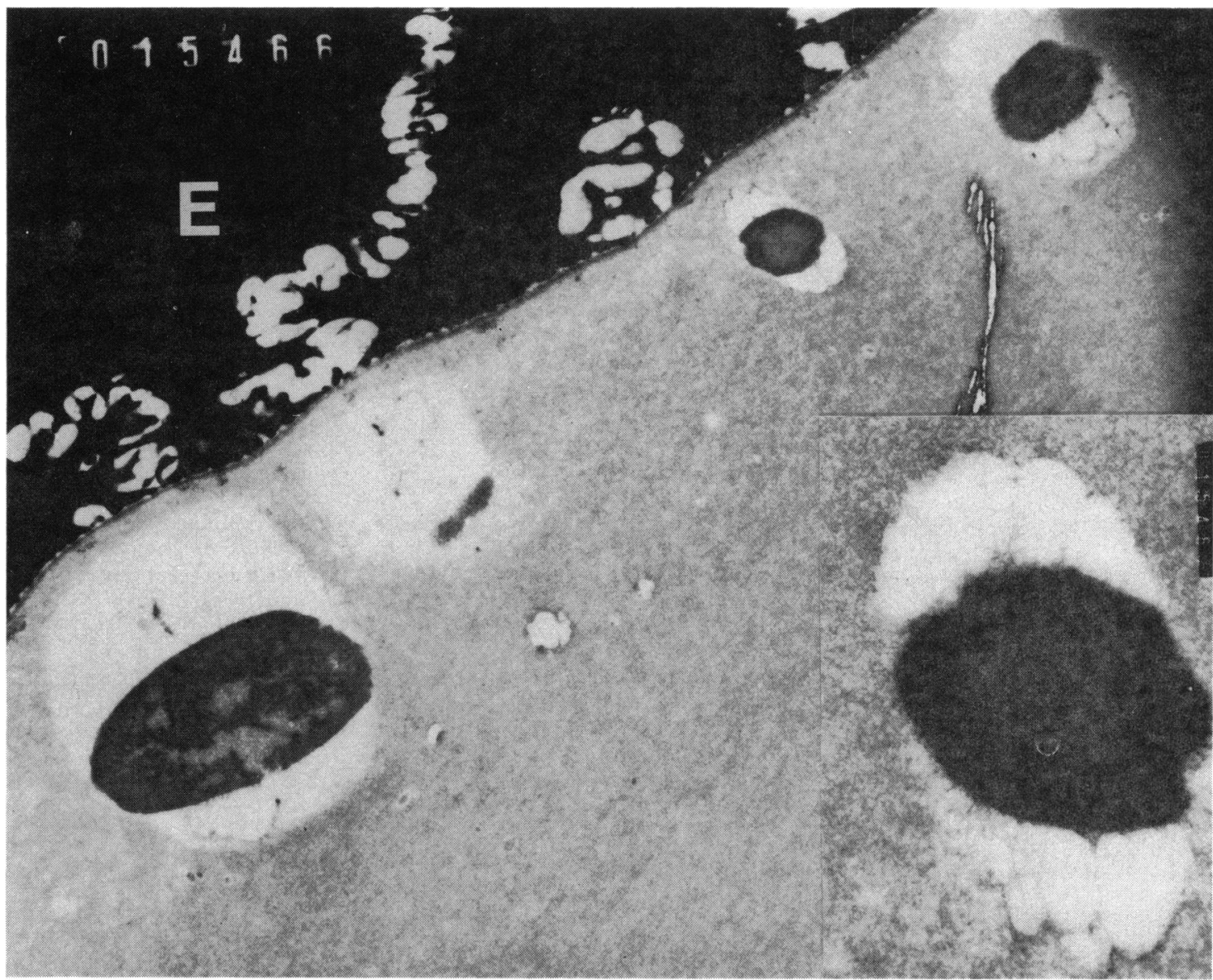

Fig. 3 Patient 1. Electron dense bodies surrounded by rarefied or vacuolated basement membrane-like granular material lie close to the basement membrane. (EM, $\times 2025)$. Inset: Detail of one of the bodies showing coalescing vacuoles. $(E M, \times 7000)$.

duced by reflection at the interfaces of a thin film. The path difference in the beams reflected from the 2 surfaces results in a difference in phase. It can be shown that when the thickness of the film approaches the wavelength of light the 2 beams will be in phase for some wavelengths and out of phase for others which hence suffer interference. ${ }^{1}$ In this way colours arising from suppression of part of the spectrum are produced. Since only a small proportion of light is reflected at each of the film surfaces, a relatively high intensity of incident light and a poorly illuminated background are necessary for interference colours to show up strongly. Their intensity is also increased when the reflectors are 'stacked' as in certain biological structures such as the 'eye' of a peacock's feather.' Multilaminar interference of this type may be responsible for the brightly coloured particles seen in the lens in myotonic dystrophy. ${ }^{2}$
In seeking the ultrastructural basis for the reflective corneal blue streaks noted in this study attention is naturally directed to the layers of basementmembrane-like material which underlie the epithelium. These layers are of alternating density and rarefaction and hence probably have different refractive indices. Although mostly parallel to the corneal surface, they are folded in places. The laminae vary considerably in thickness, but the width of many of them are of the order of the wavelengths of visible light, so that multilaminar interference giving rise to iridescence may well occur under such conditions. ${ }^{3}$ This admittedly is speculative, since both the refractive indices and the in-vivo thickness of the layers are unknown and cannot be calculated from microscopical sections. Artefactual changes doubtless occur in preparation, since Lythgoe ${ }^{4}$ noted that considerable changes in thickness of the structures 
responsible for corneal iridescence in teleost fish were brought about by the fixation and embedding processes required for electron microscopy.

The origin and significance of the curious basophilic and electron dense bodies usually found in the most superficial lamellae of the subepithelial fibrillogranular material in the first patient are unknown. Their dimensions would appear to be too small to account for the blue streaks and lines seen clinically. The possibility that they have been extruded from the epithelial cells during debridement cannot be excluded, but it is of interest that they have not been noted previously.
Mr Ron Cousens, chief photographer of the Charles Clifford Dental School, Sheffield, was responsible for the photomicrographs.

This study was aided by a grant from the National Committee for the Prevention of Blindness.

\section{References}

1 Wright WD. The rays are not coloured. New York: American Elsevier Publishing Company, 1967.

2 Dark WJ, Streeten BW. Ultrastructural study of cataracts in myotonia dystrophica. Am J Ophthalmol 1977; 84: 666-74.

3 Land MF. The physics and biology of animal reflectors. Prog Biophys Molec Biol 1972; 24: 75-106.

4 Lythgoe JN. The structure and function of iridescent corneas in teleost fishes. Proc R Soc Lond (Biol) 1975; 188: 437-57. 